Wenn europäische Unternehmen sich nicht an diese gesetzlichen Änderungen halten und ihre Systeme zur Verarbeitung von Kundendaten gegen die Grundverordnung verstoßen, kann sie das teuer zu stehen kommen: die Geldstrafen betragen $4 \%$ des Gesamtumsatzes, beziehungsweise bis $\mathrm{zu} 20$ Millionen Euro. Am Beispiel von Facebook haben sie gelernt, dass die Missachtung der Nutzerrechte ihren digitalen Ruf schädigen kann. Die schwachen Erfolge der Verbraucherorganisationen im medialen Zeitalter (bezogen auf Radio und Fernsehen) haben bereits die Reichweite und die Bedeutung der Staatsbürgerschaft und ihrer beginnenden Ausübung im Netz geändert. Die Anführer des E-Kapitalismus, die durch den Gang an die Börse schnell große Gewinne gemacht hatten, verloren im Juli 2018 zwischen 20 und $25 \%$ ihrer Aktienvermögen an der Wall Street. Daraufhin begannen sie, genau wie Facebook und Twitter, für eine klare Unterscheidung zwischen zuverlässigen und gefälschten Nachrichten einzustehen, um den Nutzern wieder Sicherheit zu suggerieren. Nachdem im April desselben Jahres der Datenskandal um Cambridge Analytica bekannt wurde, bestand die Notwendigkeit, der eigenen Diskreditierung schnellstmöglich entgegenzuwirken. Damals wurden zig Millionen von Nutzerdaten in sozialen Netzwerken zwecks der TrumpKampagne zweckentfremdet (»Facebook s'effondre de $18 \%$ à l'ouverture de Wall Street« 2018).

\title{
Sollen tausende Silicon Valleys blühen?
}

Während die transnationale Kommunikation und soziodigitale Netzwerke nationale Grenzen verschwimmen lassen, betreffen sie spezifische Regionen auf unterschiedliche Weise. Sie artikulieren sich durch wirtschaftliche und politische Auseinandersetzungen, die nicht elektronisch ablaufen. Sie bringen Bürgern in Ländern ohne fortschrittliche Technologiepolitik, darunter Lateinamerika, in eine unsicherere Lage; im Gegensatz dazu geben sie Bürgern aus europäischen Ländern mehr Möglichkeiten oder schützen sowohl deren Interessen, als auch die der dort ansässigen Unternehmen. Diese Unterschiede sind für die Diskussion in diesem Buch besonders interessant: es geht um die Reorganisation soziokultureller Aspekte der Staatsbürgerschaft. Das Fortbestehen ethnischer, nationaler und regionaler kultureller Entwicklungen, welche die angebliche Universalität des Tech-Kapitalismus widerlegen, stellt die globale Datenwirtschaft vor unterschiedliche Herausforderungen. Die lateinamerikanische Kritik am zivilisatorischen Anspruch 
des E-Kapitalismus - Zugang für alle, horizontale Interaktion, autonomes Unternehmertum mit freier Konnektivität - kann sich von den Debatten in den Metropolen inspirieren lassen und gleichzeitig eigene Handlungswege finden.

Für einen Einstieg in diesen Prozess ist eine Beobachtung der Nanostrategien der Technologieunternehmen nützlich. Wir sprechen bereits von "semigeheimen Vereinigungen « der emanzipatorischen und der dominanten Hackergemeinschaft. Es ist heutzutage bekannt, dass der Tech-Liberalismus nicht entortet ist und auch nicht völlig versteckt abläuft, sofern er seinen steuerlichen Verpflichtungen nachkommt.

Die Hauptlabore der digitalen Industrie befinden sich im Silicon Valley. Hier konzentrieren sich die Start-Up-Unternehmen, Tempel der »Flexibilität« und der »Zusammenarbeit«, die »die kreativen Beiträge jedes Einzelnen« brauchen. In der Umgebung von San Francisco stimmen Datenspeicherung und -verarbeitung mit einer expansiven Logik des kapitalistischen Liberalismus überein. Der Glanz des Silicon Valley ist nicht nur wirtschaftlicher Natur. Der Wunsch, Teil der Ideologie solcher Unternehmen zu sein, hat sich seit den ersten Jahrzehnten des 21. Jahrhunderts verbreitet. Junge Menschen wollen Silicon Valleys, Täler des Wissens, auf allen fünf Kontinenten gründen. Trotz der Ungleichheit und der schutzlosen Prekarität, die wir im Kapitel über junge Kreativschaffende und soziale Bewegungen unter der Beteiligung Jugendlicher analysiert haben, beruft sich diese so genannte "Silikonisierung" weiterhin auf »egalitäre Werte« von »visionären Start-Up-Angestellten « und der »unabhängigen Freiberufler«. Als öffentlich-private Arbeitsgemeinschaften programmierte Industrieparks und Gründerzentren beugen sich vor diesem freundlich anmutenden Kolonialismus: Er vereint die freiwillige SelbstSilikonisierung oder »Sili-Kolonisierung«, wie Eric Sadin sie nennt.

Damit wären wir auf der anderen, versteckten Seite des »Zeitalters der Zugangsmöglichkeiten « angekommen, das in den ersten Jahren des Internets so gelobt wurde. Es geht nicht mehr nur um die Erfassung und die Verwaltung von Daten. Die Begleitung unserer Existenz und unseres Zusammenlebens durch Algorithmen macht die künstliche Intelligenz »zu einer Form des ÜberIchs, das zu allen Momenten mit der Intuition der Wahrheit ausgestattet ist und das den Kurs unserer individuellen und kollektiven Handlungen auf ein Optimum lenkt « (Sadin 2018, 37). Durch die Gestaltung unseres kognitiven Horizonts ohne eine öffentliche Debatte kommt es zu einer »Schwächung der Möglichkeit zum politischen Handeln im Sinne einer a priori freiwilligen Be- 
teiligung von Einzelpersonen für das Leisten eines Beitrags zum Aufbau des Gemeinwohls« (Ibid., 38).

Wenn das Internet und die digitalen Server nicht universell sind, dann liegt das daran, dass ihre Macht zur Erfassung von Daten zur sozialen Überwachung Teil des Disputs zwischen den USA und China ist. Darin geht es um die zukünftige Hegemonie und die Expansion einer bestimmten Lebensweise. Besonders deutlich wird das beim Betrachten des Handelskriegs zwischen den beiden Ländern und dem Streit über die neue Reichweite von Huawei Mitte 2019. Die US-Regierung urteilt, dass chinesische Mobiltelefone und Tablets eine Gefahr für ihre nationale Sicherheit darstellen und hindert USUnternehmen daran, ihnen Komponenten oder Software zu verkaufen. Es geht nicht nur um einen Kampf zwischen zwei Tech-Giganten. Googles Forderung an Huawei, keine Service-Updates von Google auf Geräten von Huawei zu installieren, ist für die die asiatischen Geräte ebenso ein Schlag wie für die Google-Software Android und Gmail. Ähnlich verhält es sich bei Nutzern im Westen, die nicht wissen, ob sie mit gutem Gewissen auf 5G-Netze zugreifen können, deren Marktführer Huawei ist. Mehrere Partnerunternehmen (Alphabet, Skyworks und Xilinx) verzeichnen Preiseinbrüche. Am wichtigsten ist für uns dabei der mangelnde Schutz für Smartphone-Nutzer. Sie müssen nicht nur damit rechnen, dass ihre Daten von einer auf die andere Seite dieser Auseinandersetzung entführt werden, sondern dass sie auch über Gmail entwendet werden können, einem E-Mailanbieter mit etwa einer halben Milliarde Nutzer.

Sadin erklärt, dass seine Bedenken bezüglich der Datennutzung und der Bürgerrechte sich aus der Idee des europäischen Humanismus nähren. Es ist anzuzweifeln, dass dieser Humanismus eine solche Anerkennung für auf »der Kraft des menschlichen Verstandes« basierende »juristisch-politische Errungenschaften« verdient. Seine Glaubwürdigkeit ist des Weiteren fraglich, weil er »das grundlegende Recht auf Widerspruch und auf die Erhaltung unserer Gefühlswelt, die uns ausmacht« aufrechterhält (Ibid., 38). Nichtsdestotrotz erweist sich Sadins Diagnose über die Allianz zwischen »der technisch-wissenschaftlichen Forschung «, »dem überaus abenteuerlichen und eroberungsfreudigen Kapitalismus« und den neoliberalen Regierungen, die sich der Algorithmierung des Sozialen verschrieben haben, als interessant.

Wenn der Ortungsdienst Waze in Mexiko-Stadt, Buenos Aires oder Frankfurt meine Unsicherheiten aus dem Weg räumen kann, dann scheint es, als übersteige sein computerbasiertes Urteilsvermögen, und damit seine Dienstleistung, lokales Wissen oder zeitliche Schwankungen. Diese können 
wir Menschen nicht vorhersehen, noch weniger in einem uns fremden Land: wir können nicht im Voraus wissen, dass der Verkehr heute wegen eines großen Nachbarschaftsfest, einer Demonstration oder der Straßenarbeiten ins Stocken kommt. Delegiere ich meine Entscheidungen also an diese mir überlegene Intelligenz, die sekundengenau und multilokal arbeitet? Google und andere globale Enzyklopädien deuten das Bild einer lückenlosen, kontrollierten und im Sekundentakt kommunizierbaren Welt an.

Auf diese Weise bildet sich folglich ein »unbeabsichtigter Vorgang des Delegierens, der bewusst oder unbewusst durch die berauschende Gewohnheit des >technologischen Rituals < erregt wird, und das auf >intuitive Systeme auf eine Art Parallelmenschheit ausgerichtet ist, die auf das sgute Benehmen dieser Welt hinarbeiten soll«, so Eric Sadin (2017, 26-27). Dadurch richtet sich eine »roboterartige Verwaltung der Existenz ein, die durch hellseherische und empathische Agenten garantiert wird« (Idem).

Was sind die Konsequenzen für die Beziehungen der Bürger mit den öffentlichen Befugnissen? Die "politische Macht auf der Grundlage der Debatte und der Verpflichtung zu selbstständigen Entscheidungen beginnt zu bröckeln, und sie führt allmählich dazu, die Verantwortung für die Durchführung und Entscheidung öffentlicher Wahlen den statistischen Ergebnissen und den algorithmischen Projektionen zu überlassen« (Ibid., 30). Zahlen aus dem Jahr 2012 zeigten, dass bereits $51 \%$ des Internetverkehrs von nicht-menschlichen Entitäten ausging: Hacking-Programme und automatisierte Spamnachrichten. $20 \%$ dieser $51 \%$ wurden als »neutral« bewertet, während $31 \%$ schädlich waren. ${ }^{1}$

Weil digitale Informationen so hellsichtig und empathisch wirken, lenken sie uns von den eigentlichen Gefahren ab. Sie verleiten uns dazu, das neue Design einer App interessanter zu finden als Anlässe zum politischen Protest im digitalen Zeitalter. Wir sind eher dazu geneigt, Geräte zu bedienen, als Güter zu produzieren. Wir delegieren immer häufiger an scheinbar magische Mächte. Ich frage mich: Werden diese Trends nicht Teil des zivilisatorischen Prozesses selbst, der außergewöhnliche »Führungspersönlichkeiten« einsetzt, die das zustande bringen können, was Parteien, Gewerkschaften und soziale Bewegungen versäumen? Dahingehend sollten bestimmte empirische Vorgänge untersucht werden. Denn es gibt keinen Grund zur Annahme, dass eine derart generelle Hypothese unter gleicher Gültigkeit die aktuellen Geschehnisse in Brasilien, in den USA, in Mexiko und in einige Ländern 
Osteuropas erklären kann. Man erzählt uns auf der ganzen Welt, Lohnerhöhungen seien ebenso wie die Vermeidung unsicherer Arbeitsverhältnisse unmöglich, und zwar aufgrund der Globalisierung, der Schuldentilgung, der internationalen Abkommen und der Natur eines Marktes, von dem wir ohnehin nicht wissen, in welche Richtung er gelenkt wird. Scheint dann die nicht die Idee attraktiv, auf Geolokalisatoren zu vertrauen? Durch sie würden wir unsere Entscheidungen nicht nur an Geräte der künstlichen Intelligenz delegieren, sondern auch an Herrscher, die uns im Gegensatz zu den Schreckensmethoden der alten Tyrannen zum Glauben an Videokameras, an Zentren für künstliche Intelligenz und an Detektions- und Überwachungsgeräte bekehren, die von den Supermächten kommen.

Es ist nicht leicht, zu den soziokulturellen Folgen und den Politiken, die mit der automatisierten Organisation der Welt im Konflikt stehen, Stellung $\mathrm{zu}$ beziehen. Wir haben so viele Beweise für die Vorteile eines induzierten und hypervernetzten Lebens sowie für die Übernahme unserer Fähigkeiten als Verbraucher, Nutzer und Bürger. Im Netz gibt es zahlreiche Apps, die uns jederzeit bei der Erweiterung unserer kognitiven und kommunikativen Fähigkeiten behilflich sein können, während sie unsere Fotos und Bewegungen aufzeichnen und unsere Produktsuche und Käufe auswerten, ohne dass wir wirklich etwas kaufen. Sie sind dank einer Kreuzung aus Gesichts- und Bildschirmerkennung und unseren übrigen Präferenzen in der Lage, unsere potenziellen Interessen in einer Stadt vorauszusehen, in der wir vorher noch nie gewesen sind, zum Beispiel ob wir zu einem Kongress oder einer Sportveranstaltung gehen.

Der niederländische Experte für Cybersicherheit Victor Gevers entdeckte im Februar 2019, dass die chinesische Regierung über die Nutzung von Datenbanken der Firma SenseNets Vision Technology die Bewegungen von 2.5 Millionen Menschen in Xinjiang kontrollierte, da sie angeblich besorgt um die Uiguren und andere muslimische Minderheiten in der Bevölkerung war. Die Datenbank erhielt eine Liste von GPS-Standortmarkierungen. In 24 Stunden sah er, wie sich die überwachten Einzelpersonen durch verschiedene markierte Standorte bewegten - eine Moschee, ein Internetcafé, ein Hotel. Gevers von der GDI Foundation konnte, wie jeder andere, der es versucht hätte, auf diese Informationen (damals 6.7 Millionen Bewegungen) zugreifen und hätte sie auch manipulieren, ausspionieren oder löschen können, da die Seite nicht passwortgeschützt war. Er schickte eine Nachricht an SenseNets mit Hauptsitz in Shenzhen, um die Firma auf ihre Sicherheitslücke aufmerksam zu machen. Das Unternehmen antwortete nicht, aber schloss den Zugriff auf 
die Datenbank, sodass nun kein Eindringen außerhalb des chinesischen $\mathrm{Cy}$ bernetzes mehr möglich ist. China erklärte, dass dieses Kontrollsystem mit einem Netzwerk an Umerziehungslagern verknüpft ist und argumentiert, dass es seit dem Aufbau solcher "politischen Trainingslager« keine neuen Zusammenstöße zwischen ethnischen Gruppen gegeben habe.

Mir fallen mehrere Unterschiede zwischen dem beschriebenen Überwachungssystem und dem Öffnen von Google oder dem Bestellvorgang auf Amazon von Nutzern in lateinamerikanischen Ländern ein. Angesichts der Tatsache, dass die Geräte, beziehungsweise die Ziele der Tech-Unternehmen nicht durchschaubar sind, gestaltet sich eine Antwort auf die Unterschiede zwischen den ethnischen Gruppen in Xiajiang und unseren Netzwerken zur digitalen Umerziehung jedoch schwierig. Wir können nicht genau wissen, wie lange diese Phase andauern wird.

Sadin stellt sich die Frage, wie diese Risiken einzuschätzen sind, wenn die robotisierte und globale Gouvernementalität als »ein als nicht-politisch deklariertes und unpersönliches Projekt erscheint, das aber auch expansiv und strukturierend wirkt« (Ibid., 138). Ihm zufolge neigt die algorithmische Gouvernementalität zur Regulierung des sozialen Bereichs für eine Vermeidung jeglicher Art der Spannung. Ihre Fähigkeit, sich zwischen die Menschen zu stellen, auch zwischen Menschen und Dinge, zwischen die Dinge selbst und Organisationscodes, erzeugt eine Art »universelle Passform«der »Anpassung« zwischen jeder verbundenen Einheit, bis hin zum Bewirken einer sozialen Glättung. Diese Konfiguration erfüllt nicht mehr den von Kant angesprochenen »ewigen Frieden« oder das hegelsche »Ende der Geschichte«, sondern es manifestiert den zeitgenössischen unpersönlichen Willen zur bestmöglichen Abstimmung in der Aktion zwischen jeder organischen und materiellen Einheit (Sadin 2018, 138).

Ich sehe die Notwendigkeit, diese aus den Metropolen stammende Vision der algorithmischen Gouvernementalität mit anderen lateinamerikanischen Prozessen zu vergleichen - sozioökonomische, politische und kulturelle - die zur Entbürgerlichung führen. Es fällt schwer, diese zunehmend geglätteten Gesellschaften in ausgezehrten Ländern zu erkennen. Durch Korruption in Parteien und anderen sozial repräsentativen Institutionen sind sie kurzgeschlossen. Kriminelle Organisationen verwenden Hightech-Geräte nicht für die Erhaltung der Ordnung oder deren Transformation, sondern um sie zu brechen. Das wiederholte Versagen der auf beiden Seiten technologisch unterstützten »Antidrogenkriege« oder die prekären Versuche der sozialen Teilhabe und der kollaborativen Wirtschaft sollten auch in diese Analyse mitein- 
gebunden werden. Wir sprechen von einem Abriss des Sozialen, zusammen mit der Rationalisierung der Algorithmen.

Um die Ausbreitung der Sili-Kolonisierung nachzuweisen, sammelt Sadin Hinweise auf argentinische, brasilianische, chilenische, afrikanische und asiatische Programme, die den Markenwert Kaliforniens nutzen. High-TechUnternehmen und Inkubatoren für Startups in São Paulo führten dazu, dass die brasilianische Stadt jetzt als das »Silicon Valley Südamerikas« gilt. Santiago de Chile hat angekündigt, dem brasilianischen Beispiel folgen zu wollen. Zahlreiche lateinamerikanische Universitäten und Regierungen, die im ersten Jahrzehnt des 21 . Jahrhunderts die Ideologie des Unternehmertums als Schritt gegen Massenprekarität und Jugendarbeitslosigkeit übernommen hatten, beschließen im zweiten Jahrzehnt den »Ausstieg « aus diesem und folgen der Tendenz zur technologischen Innovation. Ein bedeutender Fall, den Sadin nicht erwähnt, ist Guadalajara im mexikanischen Bundesstaat Jalisco. Die Behörden fördern ein Programm zur Vernetzung großer Unternehmen, die meisten von ihnen US-amerikanisch, mit kleinen und mittelständischen Unternehmen. Von ihnen gibt es schätzungsweise 600 in Jalisco. Ziel des Programms ist die Gründung eines Technologie- und Kreativsystems. Dadurch sollen, wie an anderen Orten auch, Investitionen angezogen, hochwertige Arbeitsplätze geschaffen und digitale Dienstleistungen bereitgestellt werden, die diese Stadt zur zum »Guadalajara Valley« machen würden. Darüber hinaus versuchen die Behörden, die von Trump auferlegten Beschränkungen gegen Einwanderer auszunutzen. Denn unter ihnen befinden sich wohlmöglich auch einige qualifizierte Techniker, die sich dann zusammen mit den Unternehmen in Guadalajara niederlassen könnten. Man nimmt an, dass 85.000 Bewohner im Süden San Franciscos dann in Gefahr wären, ihre speziellen $\mathrm{H}-1 \mathrm{~B}-\mathrm{Visa}$ zu verlieren. Die Regierung von Jalisco lädt Experten zu den Themen Virtual Reality, Robotik und Datenanalyse nach Guadalajara ein und lockt mit Steuervorteilen und großer Medienpräsenz in Mexiko und Kalifornien. Mehrere Hochschuleinrichtungen in der Region zeigen Bereitschaft zu einer Zusammenarbeit (ITESO, ITESM, Universidad de Guadalajara, und weitere). Analysten und Befragte dieser Institutionen weisen darauf hin, dass der Diskurs in der Regierung sich nicht mit den Konflikten der städtischen Unsicherheit befasst - Guadalajara zählt zu den gefährlichsten Städten Mexikos. Gleiches gilt für den Schutz persönlicher Daten von Nutzern in Netzwerken, sowie für den Schutz akademischer Diskussionsthemen in dieser Region (Riquelme 2018; Soto 2018). Was verstehen wir von dieser Situation, wenn wir den nicht-technologischen Zusammenhängen keine Chance gewähren, ihr 
Gewicht zeigen? Sowohl die Diskriminierung von Einwanderern in den USA, als auch die mafiösen Machenschaften in Jalisco (Anfang 2019 neun Morde pro Tag) bezeugen, dass die technikbesessenen Narrative mit dem sozialen Unvermögen der Gouvernementalität verflochten sind.

Eric Sadin widmet ein Kapitel seines Buchs mit dem Titel Die SiliKolonisierung der Welt der Beschreibung der Begriffe »Brutalität« und »Kriminalität« im Sinne von Notwendigkeiten für die Entwicklung der künstlichen Intelligenz. Er unterscheidet in diesem "neofeudalen" System zwischen »vier Kasten«. Die erste sind die so genannten Königs-Codierer, also Personen mit äußerst hohen Qualifikationen in der Mathematik und im Programmieren. Sie sind in der Lage, Projekte zu definieren, die die erforderlichen technischen Anforderungen zusammenfassen und Lösungen anbieten. Eine größere und vielfältigere Kaste bildet die zweite Gruppe, die in den Forschungsabteilungen, im Design, im Marketing, den Public Relations und der Programmierung zu finden ist: Sie befinden sich nicht innerhalb des Unternehmens, sondern »im Schoß einer Atmosphäre«. Sie können in der Kantine kostenlos gesunde Gerichte essen und teilen sich ihr Mittagessen auf großen Holztischen, da dies den informellen Austausch fördert, den sie dann in Freizeiträumen und in rund um die Uhr geöffneten Fitnessstudios fortsetzen.

Es gibt eine dritte Kaste, die »auf einer Basis des Lumpenproletariats besteht und von Firmen angestellt wird, die Hardware herstellen«. Die meisten dieser Firmen sitzen in Asien, darunter die taiwanesische Gruppe Foxconn. Es handelt sich um Montagewerke mit beklagenswerten sanitären Verhältnissen, in denen ohne Rücksicht auf ökologische und soziale Auswirkungen Rohmineralien verarbeitet werden.

Die vierte Kaste ist die der in der so genannten plattformbasierten Wirtschaft tätigen Personen: Uber-Fahrer und Arbeiter bei ähnlichen Unternehmen, Immobilienvertreter. Scheinbar unabhängige Dienstleistungsanbieter ohne Rechte, die unmittelbar mit Computersystemen verbunden arbeiten. Diese qualifizieren sie, fördern sie oder setzen sie massiv unter Druck, und zwar immer mit einem enormen Gewinnanteil für das Unternehmen.

Die Einschränkung dieser Sadin-Analyse besteht darin, dass sie von einem Prozess innerhalb oder zwischen Unternehmen ausgeht. Dabei sieht sie keine Widersprüche, Hierarchien und keine Ausbeutung bei den sozialen Bedingungen der unterschiedlichen geoökonomischen und geopolitischen Regionen, wie sie beim Kontrast zwischen Kalifornien und Guadalajara angedeutet wurden. Sadin stellt in seiner kurzen Analyse des Silicon-Valley Pro- 
pagandastils etwas ähnliches fest: zusätzlich zur Förderung ihres libertären, kreativen und vermittelnden Geistes, etwa auf TED-Konferenzen (Technology, Entertainment and Design), auf Veranstaltungen und in Zeitschriften, begreifen diese Akteure ihre Aufgabe als »Mission« mit dem Ziel der »Erhöhung des Lebensstandards durch Technologie « und sehen ihre Führungskräfte als fast heiligengleiche Vorbilder, wie Steve Jobs sich sogar selbst bezeichnete. Der französische Philosoph weist darauf hin, dass der Begriff »Technology Evangelist « nicht zufälligerweise eine Arbeitsposition in der digitalen Industrie bezeichnet. Hararis Beschreibung des Dataismus als »Religion der Daten«bestärkt diese Interpretation. Aber es ist notwendig, dieses expansive und erlösende Vorstellungsbild auf der Basis empirischer Untersuchungen zu folgern. Carmen Bueno, Gustavo Lins und Luis Reygadas wurden in diesem Buch bereits vorgestellt. Durch diese Studien haben wir die Möglichkeit zu verstehen, wie die Köderwirtschaft und die Selbstausbeutung mit der einvernehmlichen politischen Unterwerfung verflochten sind.

\section{Wie Bürger sich informieren}

Die kombinierte Analyse zwischen Online- und Offlinewelt, den diskursiven Strategien der Technikbranche und deren wirtschaftlichen Strukturen auf nationaler und transnationaler Ebene ist im Kontext der Rezeption und der Nutzerrechte zu einem Merkmal der fortgeschrittenen Kommunikationsforschung in Lateinamerika geworden (Crovi und Trejo 2018). Die erwähnten Aspekte zur Kontinuität zwischen digitalisierten Arbeitsmärkten und ihren soziopolitischen Kontexten wird auch in den Formen des Zugangs und der Aneignung kultureller Güter sichtbar. So expansiv die digitale Welt auch ist: Wir bilden und informieren uns über die Geschehnisse in der Gesellschaft anhand schriftlicher Medien, audiovisueller Inhalte, über das Internet, und, nicht zu vergessen, über den persönlichen Austausch zu zweit oder in Gruppen, von Angesicht zu Angesicht. Es ist bekannt, dass die Schule eine entscheidende Rolle bei der Bildung des Menschen zum Bürger spielt. Doch genau wie Pädagogen in den 1970er-Jahren das Fernsehen als Feind sahen, widersetzt sich die Schule seit Jahrzehnten dem Einzug digitaler Ressourcen in den Unterricht. Den Pädagogen von heute fällt es schwer zu akzeptieren, dass deren breite Informationskapazität fast immer mit einem Unterhaltungswert gekoppelt ist. Bildung und Staatsbürgerschaft sind ernste Angelegenheiten. 